\title{
BMJ Open Cohort profile: a nationwide population- based retrospective assessment of oesophageal cancer in the Finnish National Esophago-Gastric Cancer Cohort (FINEGO)
}

\begin{abstract}
Henna K Söderström, ${ }^{1}$ Jari Räsänen, ${ }^{1}$ Juha Saarnio, ${ }^{2}$ Vesa Toikkanen, ${ }^{3}$ Tuula Tyrväinen, ${ }^{4}$ Tuomo Rantanen, ${ }^{5}$ Antti Valtola, ${ }^{5}$ Pasi Ohtonen, ${ }^{2}$ Minna Pääaho, ${ }^{2}$ Arto Kokkola, ${ }^{6}$ Raija Kallio, ${ }^{7}$ Tuomo J Karttunen, ${ }^{8}$ Vesa-Matti Pohjanen, ${ }^{8}$ Ari Ristimäki, ${ }^{9,10}$ Simo Laine, ${ }^{11}$ Eero Sihvo, ${ }^{12}$ Joonas H Kauppila (1) ${ }^{2,13}$
\end{abstract}

To cite: Söderström HK, Räsänen J, Saarnio J, et al. Cohort profile: a nationwide population-based retrospective assessment of oesophageal cancer in the Finnish National Esophago-Gastric Cancer Cohort (FINEGO). BMJ Open 2020;10:e039575. doi:10.1136/ bmjopen-2020-039575

- Prepublication history for this paper is available online. To view these files, please visit the journal online (http://dx.doi org/10.1136/bmjopen-2020039575).

Received 19 April 2020 Revised 02 September 2020 Accepted 22 September 2020

D Check for updates

(C) Author(s) (or their employer(s)) 2020. Re-use permitted under CC BY-NC. No commercial re-use. See rights and permissions. Published by BMJ.

For numbered affiliations see end of article.

Correspondence to Dr Joonas H Kauppila; joonas.kauppila@ki.se

\section{ABSTRACT}

Purpose The Finnish National Esophago-Gastric Cancer Cohort (FINEGO) was established to combine the available registry data with detailed patient information to form a comprehensive, retrospective, population-based research platform of surgically treated oesophageal and gastric cancer in Finland. This cohort profile describes the 2045 surgically treated patients with oesophageal cancer included in the FINEGO cohort.

Participants Registry data were collected from the National Cancer, Patient, Education and Death Registries from 1 January 1987 to 31 December 2016. All patients over 18 years of age, who had either curative surgery, palliative surgery or salvage surgery for primary cancer in the oesophagus are included in this study.

Findings to date 2045 patients had surgery for oesophageal cancer in the selected time period. $67.2 \%$ were man, and the majority had only minor comorbidities. The proportions of adenocarcinomas and squamous cell carcinomas were $43.1 \%$ and $44.4 \%$, respectively, and $12.5 \%$ had other or missing histology. Only about $23 \%$ of patients received neoadjuvant therapy. Oesophagectomy was the treatment of choice and most patients were treated at low-volume centres, but median annual hospital volume increased over time. Median overall survival was 23 months, 5-year survival for all patients in the cohort was $32.9 \%$ and cancer-specific survival was $36.5 \%$.

Future plans Even though Finland only has a population of 5.5 million, surgery for oesophageal carcinoma has not been centralised and therefore previously reported results have mostly been small, single-centre cohorts. Because of FINEGO, we now have a population-based, unselected cohort of surgically treated patients, enabling research on national trends over time regarding oesophageal cancer, including patient characteristics, tumour histology, stage and neoadjuvant treatment, surgical techniques, hospital volumes and patient mortality. Data collection is ongoing, and the cohort will be

\section{Strengths and limitations of this study}

- The main strengths include the population-based design with complete and accurate ascertainment of all surgically treated patients with oesophageal cancer in Finland, mitigating selection bias.

- Complete follow-up is a strength.

- The sample size is large enough to enable multivariable analysis, and analyses in smaller subgroups of patients.

- Exclusion of non-surgically treated patients and the information lag of up to 2 years are the main limitations.

expanded to include more detailed data from patient records and national biobanks.

\section{INTRODUCTION}

Oesophageal carcinoma is an aggressive disease with poor prognosis. According to the European Cancer Registry based study on survival and care of cancer patients-5 (EUROCARE-5) study, only about $10 \%$ of patients are alive 5 years after being diagnosed. ${ }^{1}$ Cancer stage strongly influences oesophageal cancer survival, and unfortunately, the majority of patients have local $(30 \%)$ or distant $(40 \%)$ metastases at the time of diagnosis. This markedly lowers the 5-year survival from $39 \%$ in patients with the localised disease to $5 \%$ in cases with distant metastasis. ${ }^{2}$ Worldwide, it is the 12th most common cancer in terms of incidence, and the 6 th most common cause of cancer death. ${ }^{3}$ According to the WHO, about 53000 people are diagnosed with oesophageal cancer in Europe every year 2018. The incidence of oesophageal cancer is increasing 


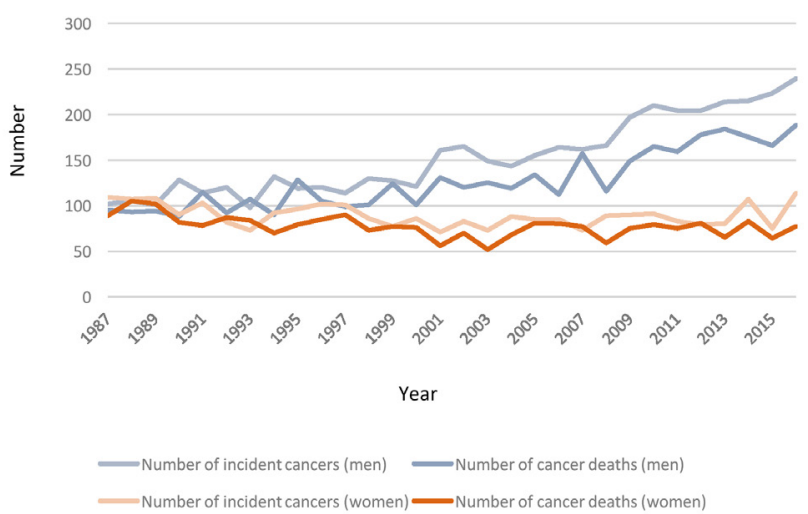

Figure 1 The number of incident oesophageal cancers and oesophageal cancer deaths by sex, according to the Finnish Cancer Registry.

in men in Finland, while it is slightly decreasing in women (figure 1). ${ }^{4}$ Of the histological subtypes, the incidence of oesophageal adenocarcinomas is increasing, ${ }^{56}$ while squamous cell carcinomas (SCC) are decreasing. ${ }^{6}$

Radical surgery combined with neoadjuvant chemo(radiotherapy) is the standard of care for locally advanced, resectable oesophageal carcinomas. ${ }^{7}$ Surgery for oesophageal cancer is a high-risk procedure with high morbidity ${ }^{8}$ and mortality. ${ }^{9}$ In early cases where the tumour is limited to the mucosa with no sign of nodal involvement, endoscopic treatment with either endoscopic mucosal resection (EMR) or endoscopic submucosal dissection (ESD), is a safe and effective option with excellent long-term results. $^{1011}$

Several studies support centralisation, showing a clear survival benefit for patients who had surgery at highvolume centres $^{12} 13$ and by high-volume surgeons. ${ }^{14} 15$ Studies have shown minimally invasive oesophagectomy (MIO) to be a feasible surgical technique in treating oesophageal carcinoma, ${ }^{16} 17$ with equal oncological results as open oesophagectomy. ${ }^{18} 19$ The number of MIOs in Europe are rising, with less than $20 \%$ of all eosophagectomies performed with the transthoracic minimally invasive approach in 2007, compared with almost $50 \%$ in $2014 .^{20}$

The nationwide registries in Finland systematically collect data on diseases, including oesophageal cancer. However, these data are limited in detail and scattered over several databases limiting the usability in surgical research as such. The Finnish National Esophago-Gastric Cancer Cohort (FINEGO) was established to retrospectively gather detailed clinical data on all surgically treated oesophageal and gastric cancers in Finland to create a nationwide population-based registry, combining registry data, patient records data, as well as biobanks, for future research purposes. ${ }^{20}$

There are several gaps of knowledge in the diagnosis and treatment of oesophageal cancer. For instance, the changing demographics of oesophageal cancer and the effects of oncological treatment and centralisation in surgical patients are not completely clear. Furthermore, the associations between anastomotic methods, surgical approach, treatment complications and short-term and long-term mortality, as well as cancer survivorship are unclear. Lastly, the large-scale histological assessment of oesophageal cancer should provide robust information on potential new predictive or prognostic histological markers.

In this cohort profile, we describe the patients with oesophageal cancer included in the FINEGO cohort.

\section{COHORT DESCRIPTION}

FINEGO is a population-based, nationwide, a retrospective cohort study of all surgically treated patients with oesophageal and gastric cancer in Finland since 1987 and onwards. The collaborative group comprises of senior consultant upper gastrointestinal or thoracic surgeons from all five academic centres, and one non-academic central hospital, conducting oesophageal and gastric cancer surgery in Finland. In addition, senior oesophagogastric pathologists, oncologists and biostatisticians are involved in the design and execution of the study.

All patients over 18 years of age, who had either curative surgery, palliative surgery or salvage surgery for primary cancer of epithelial origin in the oesophagus or stomach are included in the FINEGO cohort. Patients who were treated endoscopically, that is, with endoscopic mucosal resection or submucosal dissection with curative intent, are also included. This study presents the oesophageal cancer cases included in the cohort.

Complete information on the details of data collection, sample size calculation and data management plan have been described in the study protocol. ${ }^{20}$

\section{Data sources}

The registry data were collected from the national registries from 1 January 1987 to 31 December 2016. All healthcare units in Finland are mandated by law to enter data into these registries making them highly comprehensive. ${ }^{21}$ Registry data were combined using the immutable 11-digit personal identification number assigned to all residents in Finland. Age and sex were derived from the personal identity numbers containing this information (the first six digits is the date of birth, and the eighth digit is based on the sex of the person).

The Finnish Cancer Registry provided information on cancer, including date and type of cancer, topography (location), morphology (histology) information, cancer stage in a written format (local, locally advanced, advanced), as well as crude information on cancer treatment, including the use of surgical treatment, chemotherapy, radiotherapy and hormonal treatment (yes/no).

The Finnish Patient Registry provided information on the discharge dates, diagnosis codes and operation codes assigned for each patient during that admission, as well as the code of the healthcare unit where the admission took place. This data was used for defining cancer diagnosis, treatment (operation codes), comorbidity (diagnosis codes) and hospital volume data (operation and 
healthcare provider codes). Comorbidities were defined using the well-validated Charlson Comorbidity Index (CCI) not including oesophageal cancer, by retrieving diagnoses before but excluding the index admission for surgery. ${ }^{22}$ The annual hospital volume was assessed by calculating the number of benign and malignant oesophagectomies during the year of surgery in the hospital the patient was operated in for each patient.

Statistics Finland provided data on the dates and causes of death, as well as the highest education obtained by the patients.

\section{Patient identification}

Diagnosis numbers were searched for in the Finnish Cancer Registry and the Finnish Patient Registry to identify patients with cancer of the oesophagus (150.0-150.9 in the 8th and 9th version of the International Classification of Diseases, ICD-8 and ICD-9, and C15 in the ICD-10) as well as those with unclear oesophageal tumours (230 in the ICD-8, 235 in the ICD-9 and D37.1 and D37.7 the ICD-10). Surgical codes were then searched for in the Finnish Patient Registry to identify those undergoing surgical treatment (oesophagectomy or endoscopic mucosal surgery). Using both registries concurrently guarantees a very high probability of finding all existing cases.

\section{Statistical analysis}

Statistical analysis was done using SPSS V.21.0 (SPSS). Kaplan-Meier curves and the Life Table method were used for survival analysis. Survival was calculated from the date of surgery until death or the end of follow-up, whichever came first. Cancer-specific mortality refers to mortality related to oesophagogastric cancer, which was selected as the most appropriate to take into account the potential misclassification between oesophageal and gastric cancers. ${ }^{23}$

\section{Patient and public involvement}

Patients or public were not involved in the development of the research question and study design or conducting the present study.

\section{FINDINGS TO DATE}

Between 1 January 1987 and 31 December 2016, 2045 patients were surgically treated for oesophageal cancer in Finland (figure 2), which is higher than the initial estimate of 1800 patients. ${ }^{20}$ According to the official statistics by the Finnish Cancer Registry, a total of 7280 patients were diagnosed with oesophageal cancer 1987-2016, and the number of incident oesophageal cancer cases and cancer deaths decreased during the study period (figure 1). ${ }^{4}$ The patients in FINEGO were mostly identified in both of the registries, but a small number (6.3\%) were missing from the Cancer Registry (table 1).

At the time of surgery, the vast majority $(86.1 \%)$ of patients were between 50 and 80 years of age. The median

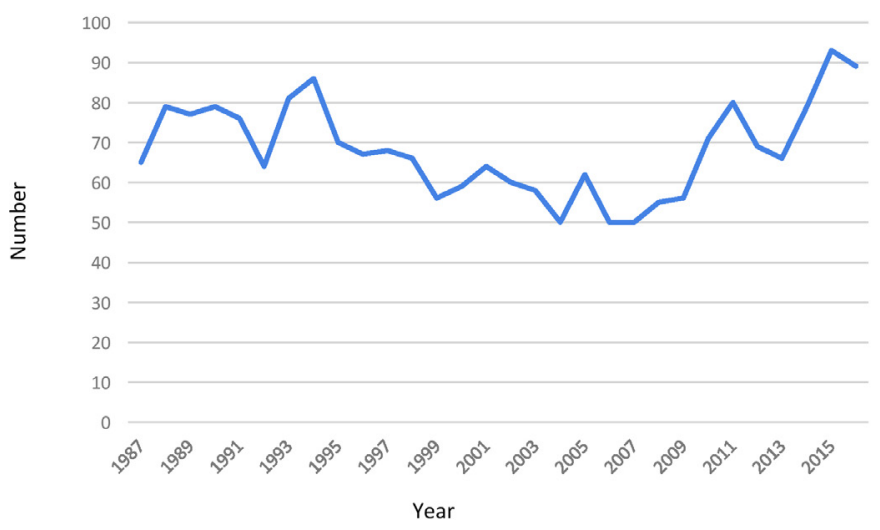

Figure 2 Number of surgically treated patients with oesophageal cancer between 1987 and 2016.

age of the patients remained quite constant over time, with a median of 65.5 years, ranging from 61 to 71 years. In the cohort, $67.2 \%(\mathrm{n}=1375)$ of the patients were man. Some $65.4 \%(n=1337)$ had a CCI of $0,22.8 \%(n=467)$ had CCI of 1 and $11.8 \%(n=241)$ had a CCI of 2 or higher (table 2). Educational data were missing for the majority of patients $(59.9 \%, \mathrm{n}=1225)$, but of those with data available 59.8\% ( $\mathrm{n}=491)$ had less than 12 years of education, while $28.8 \% \quad(n=236)$ had an undergraduate degree and only 93 patients (11.3\%) had more than 15 years of education indicative of a graduate degree (table 2 ).

A slightly larger proportion of patients had SCC (44.4\%, $\mathrm{n}=908)$ compared with adenocarcinomas $(43.1 \%, \mathrm{n}=882)$. The tumour was classified as 'other' in $6.3 \%(\mathrm{n}=129)$ of the cases and in $6.2 \% \quad(n=126)$ histological data were missing. The cancer was staged as local at the time of surgery in $28.9 \%(n=590)$ of the patients. In $18.7 \%$ (382) of the patients the cancer was staged as locally advanced, and in $18.7 \%(\mathrm{n}=382)$ as advanced. In one-third of the cases, stage was either unclear or missing. Patient demographics are shown in table 2 .

According to the information available in the Finnish Cancer Registry, the vast majority of surgically treated patients $(77.3 \%, \mathrm{n}=1581)$ did not receive neoadjuvant therapy. Neoadjuvant chemotherapy was administered to $242(11.8 \%)$ patients, while 222 patients received either chemoradiation $(n=119,5.8 \%)$ or radiotherapy alone

Table 1 Identification of the patients with oesophageal cancer by source registry

\begin{tabular}{lc}
\hline & $\begin{array}{l}\text { Patients, } \\
\text { Number (\%) }\end{array}$ \\
\hline Total & $2045(100.0)$ \\
$\begin{array}{l}\text { Cancer diagnosis in both hospital } \\
\text { discharge registry and cancer registry }\end{array}$ & $1900(92.9)$ \\
$\begin{array}{l}\text { Cancer diagnosis in only hospital } \\
\text { discharge registry }\end{array}$ & $129(6.3)$ \\
$\begin{array}{l}\text { Cancer diagnosis in only cancer registry } \\
\begin{array}{l}\text { Unclear tumour diagnosis and surgery } \\
\text { code in hospital discharge registry }\end{array}\end{array}$ & $14(0.7)$ \\
\hline
\end{tabular}


Table 2 Demographics of the surgically treated patients with oesophageal cancer in Finland 1987-2016

\begin{tabular}{lc|}
\hline & Patients, Number (\%) \\
\hline Total & $2045(100.0)$ \\
\hline Age at surgery & \\
\hline$\leq 50$ years & $178(8.7)$ \\
\hline $51-60$ years & $449(22)$ \\
\hline $61-70$ years & $750(36.7)$ \\
\hline $71-80$ years & $561(27.4)$ \\
\hline$>80$ years & $107(5.2)$ \\
\hline Sex & \\
\hline Male & $1375(67.2)$ \\
\hline Female & $670(32.8)$ \\
\hline Education & \\
\hline$\leq 12$ years & $491(24.0)$ \\
\hline $13-15$ years & $236(11.5)$ \\
\hline$>15$ years & $93(4.5)$ \\
\hline Missing & $1225(59.9)$ \\
\hline Charlson comorbidity index \\
\hline 0 & $1337(65.4)$ \\
\hline 1 & $467(22.8)$ \\
\hline 2 & $161(7.9)$ \\
\hline 3 & $58(2.8)$ \\
\hline Stage & $22(1.1)$ \\
\hline Local & $126(6.2)$ \\
\hline Locally advanced & $382(18.7)$ \\
\hline Advanced & $382(18.7)$ \\
\hline Missing & $565(27.6)$ \\
\hline Squear & $126(6.2)$ \\
\hline Missing & \\
\hline
\end{tabular}

$(\mathrm{n}=103,5 \%)$. The use of neoadjuvant therapy increased, as only $14.8 \%$ received neoadjuvant therapy during $1987-$ 2006 , while $37.6 \%$ of the patients received it during 2007 2016. Of all surgically treated patients, $84.7 \% \quad(n=1733)$ had open surgery, whereas $12.4 \% \quad(n=253)$ were operated with a minimally invasive approach. Oesophagectomy was by far the most common procedure $(\mathrm{n}=1767,86.4 \%)$, followed by gastrectomy $(\mathrm{n}=191,9.3 \%)$ and oesophagogastrectomy $(\mathrm{n}=28,1.4 \%)$. In total, 59 patients in the cohort $(2.9 \%)$ were treated with either EMR or ESD (table 3).

Median annual hospital volumes for oesophageal cancer surgery, on the other hand, did systematically and
Table 3 Treatment details of the patients with oesophageal cancer in included in FINEGO

\begin{tabular}{lc}
\hline & Patients, Number (\%) \\
\hline Total & $2045(100.0)$ \\
\hline Surgery type & $191(9.3)$ \\
\hline Gastrectomy & $1767(86.4)$ \\
\hline Oesophagectomy & $28(1.4)$ \\
Oesophagogastrectomy & $59(2.9)$ \\
\hline EMR or ESD & \\
\hline Surgical approach & $1733(84.7)$ \\
\hline Open & $253(12.4)$ \\
\hline Minimally invasive & $59(2.9)$ \\
\hline Not applicable & \\
\hline Neoadjuvant treatment & $1581(77.3)$ \\
\hline None & $242(11.8)$ \\
\hline Chemotherapy & $103(5.0)$ \\
\hline Radiotherapy & $119(5.8)$ \\
\hline Chemoradiotherapy & $535(26.2)$ \\
\hline Hospital volume of oesophagectomy \\
\hline 1-5 per year & $766(37.5)$ \\
\hline 6-20 per year & $469(22.9)$ \\
\hline Over 20 per year & $275(13.4)$ \\
\hline Not applicable &
\end{tabular}

EMR, endoscopic mucosal resection; ESD, endoscopic submucosal dissection; FINEGO, Finnish National Esophagogastric Cancer Cohort.

markedly increase over time. Between 1987 and 1999 the median annual hospital volume was 6 patients, from year 2000 to 2009 the median was 10.5 patients and in the years 2010-2016 the median had increased to 18 patients. Of all patients, $26.2 \%$ were treated in hospitals that perform $1-5$ operations per year, $37.5 \%$ in hospitals with an annual volume of $6-20$ cases and $22.9 \%$ in hospitals that perform over 20 surgeries per year (table 3 ). Of the 671 patients undergoing oesophagectomy during the last 10 years of the study period (2007-2016), only 108 (16.1\%) were treated in hospitals performing 1-5 oesophagectomies per year, while $282(42.0)$ were treated in hospitals with 6-20 oesophagectomies and $281(41.9 \%)$ were treated in hospitals with $>20$ oesophagectomies. Median annual hospital volumes and median patient age over time are shown in figure 3 .

Median overall survival (OS) was 23 months. 1-year, 3-year, 5-year and 10-year OS for all patients in the cohort were $65.1 \%, 40.7 \%, 32.9 \%$ and $23.2 \%$, respectively. At the time of follow-up, 746 patients (36.5\%) surgically treated for oesophageal carcinoma were still alive. All-cause mortality was $73.6 \%(\mathrm{n}=1506)$, of which $1299(63.5 \%)$ were oesophagogastric cancer-related according to the causes of death registry. All-cause 


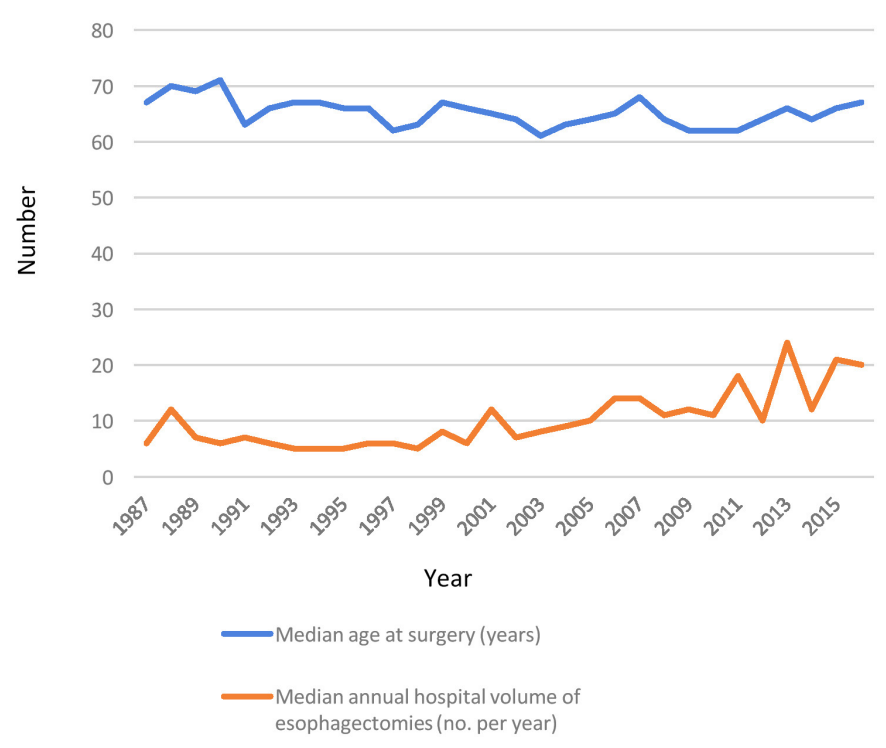

Figure 3 The median age at surgery and median annual hospital volume of oesophagectomies over time.

and oesophagogastric cancer-specific mortality are depicted in figures 4 and 5 .

\section{STRENGTHS AND LIMITATIONS}

FINEGO was established to combine the available registry data with detailed patient information to form a comprehensive, retrospective, population-based research platform of surgically treated oesophageal and gastric cancer in Finland. ${ }^{20}$ This cohort profile describes the baseline registry data of the 2045 patients with oesophageal cancer included in the FINEGO cohort.

There are many strengths to the present study, the most important ones being the size of the cohort and the extensive elimination of biases. With a total of 2045 patients, the sample size is large enough to allow for survival and regression analyses even in smaller subgroups of patients. Due to the population-based design that includes all

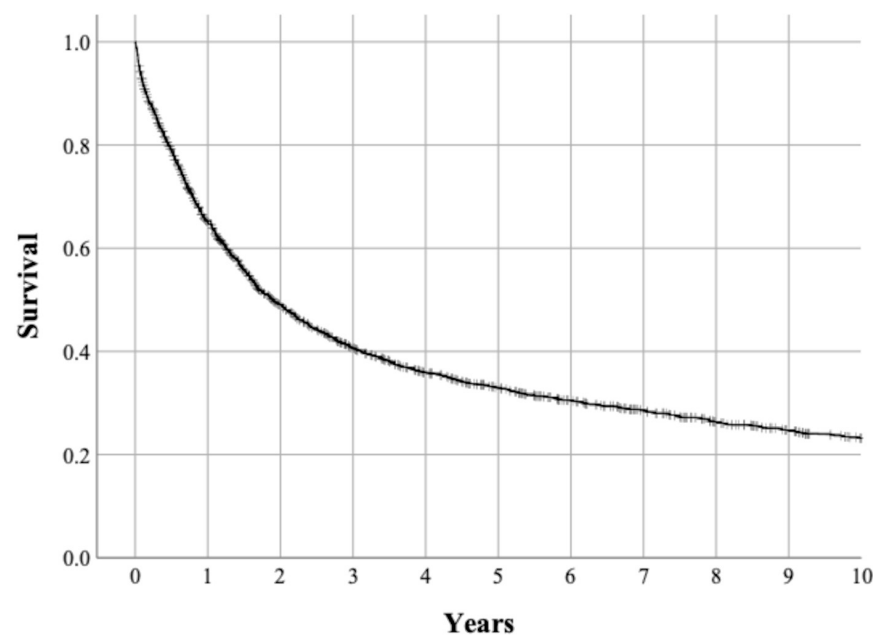

Figure 4 Kaplan-Meier curve depicting 10-year all-cause mortality in the surgically treated patients with oesophageal cancer.

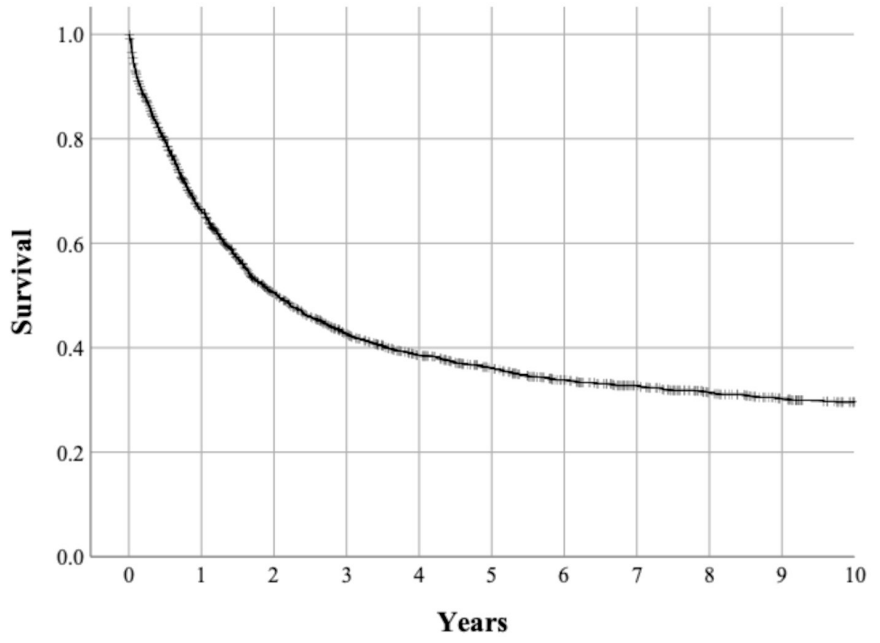

Figure 5 Kaplan-Meier curve depicting 10-year oesophagogastric cancer-specific mortality in patients with oesophageal cancer.

surgically treated patients with oesophageal cancer in Finland, selection bias is mitigated. The fact that the cohort was created using a combination of registry and patient records data reduces information bias.

Because FINEGO is a cohort comprising of reallife, unselected data on surgical patients, it is markedly different from other, global collaboratives such as the Worldwide Esophageal Cancer Collaboration ${ }^{24}$ or Esophagectomy Complications Consensus Group. ${ }^{25}$ These collaboratives typically only include expert, high-volume centres, whereas FINEGO allows for more wide-ranging conclusions and applications. The main limitations of the present study are its retrospective nature and the exclusion of all non-surgical patients. The retrospective study design potentially weakens the data quality and generates a possibility of missing data on for example postoperative complications, but on the other hand, it enables the quick establishment of a large patient cohort. To offset these potential issues, two separate high-quality databases were used. In addition, patient records are being manually collected and will be vigorously checked and validated for quality, further improving the reliability of the resulting cohort. The exclusion of non-surgical patients limits the potential of the cohort, as it excludes patients undergoing curatively intended or palliative chemotherapy and/or radiotherapy, reducing the possibilities to compare for example surgery and curatively intended chemoradiotherapy.

Some variables are not recorded in the registries and cannot be reliably retrieved from the patient records. However, the data quality and missing data will be meticulously checked before running the substudies, and the missing data will be taken into account by using multiple imputation methods in the analyses to reduce bias from missing data. Even with thorough reviewing, this cohort will not be able to provide reliable data for studying potential risk factors for oesophageal carcinoma such as alcohol and tobacco use, dietary factors, exercise habits 
and patient lifestyle or socioeconomic factors, as these are not reliably recorded in the records. Also, educational status is incomplete, with information on years of education available for only $40.1 \%$ of patients, and it is not available in the patient records. Years of education do not necessarily translate into a certain socioeconomic standard further limiting its usefulness in describing patients. Other primarily missing or potentially unreliable information, such as tumour stage at the time of operation or administered (neo)adjuvant therapy, are comprehensively recorded and can be found by manual review of patient records making these factors more useful and interesting for further substudies. Taken together, the FINEGO cohort will be most suitable for studying the effects of various surgical, oncological, pathological and surgeon-related and hospital-related factors in the surgical treatment of oesophageal cancer, while its value in evaluating risk factors of oesophageal cancer is limited.

A previous population-based study reported prognostic factors and survival in oesophageal cancer in a similar cohort in Sweden. ${ }^{26}$ The age distribution in the Swedish study was similar to the present study. In Finland, the median age of the patients did not increase over time despite the rise in life expectancy, ${ }^{27}$ but remained quite constant with a median of 65.5 years. The FINEGO cohort had a larger proportion of women, $32.8 \%$ compared with $23.6 \%$. There was a marked difference in gender distribution between patients with adenocarcinoma and patients with SCC in the Swedish cohort, and woman was a positive prognostic factor for survival in SCC. ${ }^{26}$ This interesting finding will be evaluated in future studies of the FINEGO cohort. The proportion of patients with comorbidities in this study was similar to the Swedish study.

In the present study, one-third of the patients' stage was classified as 'unclear' or 'missing'. Of all tumours, $28.9 \%$ were 'local', $18.7 \%$ 'locally advanced' and up to $18.7 \%$ were classified as 'advanced'. These stages differ markedly from several landmark studies, for example, in the ChemoRadiotherapy for Oesophageal cancer followed by Surgery Study (CROSS) trial $84 \%$ of the cases were classified as T3, ${ }^{28}$ and Low et $a l^{29}$ reported $66.6 \% \mathrm{~T} 3$ tumours when benchmarking surgical complications. Almost one-fifth of the patients having advanced disease raises concerns in the cancer registry variable reflecting tumour stage in the preoperative situation. A possible explanation is that tumour stage might have not been reported to the cancer registry preoperatively due to unclear stage, only to be reported several months or years after the initial diagnosis, that is, at the time of distant recurrence. Reflecting the distribution of tumour stage in the previous studies, those having unclear or missing tumour stage are most likely to have locally advanced disease. To assess this, the Tumor-Node-Metastasis (TNM) stages in the FINEGO cohort will be validated manually from patient records against the registry data. Furthermore, the large number of unclear stages make staging data potentially unreliable. On the other hand, a large proportion of local tumours could explain the relatively good OS.
Neoadjuvant treatment was implemented in Finland around the year 2000, and it became standard care about a decade later. This is reflected in the results, and contributes to why only about $10 \%$ of the cohort, of which $44 \%$ were SCC, received (chemo) radiotherapy which is considered standard-of-care today. However, during the last 10 years, more than one-third of the patients received neoadjuvant therapy. Potentially, there might be a lack of reporting of neoadjuvant treatment to the Finnish Cancer Registry by the clinicians when the initial cancer registry information has been already submitted at the time of diagnosis and when the decision to give neoadjuvant treatment has not yet been made. Neoadjuvant treatment will be validated from the patient files in future studies, as 'neoadjuvant' was defined as chemotherapy, radiotherapy or chemoradiotherapy with a curative intent within 4 months of diagnosis without specifics. Effect of neoadjuvant therapy on survival will be one branch for further study, as we should be able to see a change in survival over time considering that very few received preoperative treatment before the year 2000.

Even though Finland only has a population of 5.5 million, surgery for oesophageal carcinoma has not been centralised and most centres would be classified as low volume. Historically, this is due to the traditions and long geographical distances between hospitals. Median annual hospital volumes for oesophageal cancer surgery increased markedly during the study period. In the 1980s and 1990s, on average, only six patients had surgery at any given hospital each year. This number increased over time, and in the years 2010-2016 the median annual volume had tripled to 18 patients. This increase reflects governmental centralisation efforts. In the total cohort, only about $23 \%$ of the patients were treated in centres that perform over 20 operations annually, while a quarter of all patients $(26.2 \%)$ were treated in hospitals with a very low annual volume of $1-5$ operations.

According to the Finnish National Cancer Registry, 372 patients were diagnosed with oesophageal cancer in 2017, and 282 died of the disease. ${ }^{4}$ Five-year survival was $17.3 \%$ disregarding stage and treatment. ${ }^{4}$ In the FINEGO cohort, 5-year survival of the surgically treated patients was $32.9 \%$, which is markedly higher than in the unselected Cancer Registry cohort.

Because of FINEGO, we now have a population-based, unselected cohort of surgically treated patients, enabling research on national trends over time regarding oesophageal cancer, including patient characteristics, tumour histology, stage and neoadjuvant treatment, surgical techniques, hospital volumes and patient mortality. In the future, the study will be expanded every 5 years ensure that the most recent, accessible data is available for analysis. The nationwide collection of patient records and the collection of histological pre-operative gastroscopy and surgical specimen samples from national Biobanks is progressing. Once more data is gathered, several options for future research become available. Examples include assessment of the relevance of modifiable risk factors, 
such as preoperative feeding, surgical approach or type of analgesia, optimal treatment and patient prognosis, and the association between hospital volume and surgical outcomes.

In conclusion, the FINEGO collaboration will continue to collect data, creating a solid platform for future research efforts into oesophageal and gastric cancers.

\section{COLLABORATION}

All data from FINEGO presented in this article are stored by the research group on safe servers at University of Oulu, Finland, and handled confidentially. Currently, only the research team has access to the data. Researchers interested in collaboration, for example joint efforts combining the dataset with other population-based studies, are welcome to contact Joonas Kauppila (joonas. kauppila@oulu.fi),principal investigator.

\section{Author affiliations}

${ }^{1}$ Department of General Thoracic and Oesophageal Surgery, Heart and Lung Centre, University of Helsinki and Helsinki University Hospital, Helsinki, Finland

${ }^{2}$ Surgery Research Unit, Medical Research Center Oulu, Oulu University Hospital and University of Oulu, Oulu, Finland

${ }^{3}$ Department of Cardiothoracic Surgery, Heart Center, Tampere University Hospital and University of Tampere, Tampere, Finland

${ }^{4}$ Department of Gastroenterology and Alimentary Tract Surgery, Tampere University Hospital, Tampere, Finland

${ }^{5}$ Department of Surgery, University of Eastern Finland and Kuopio University Hospital, Kuopio, Finland

${ }^{6}$ Department of Surgery, University of Helsinki and Helsinki University Hospital, Helsinki, Finland

${ }^{7}$ Department of Oncology and Haematology, Oulu University Hospital, Oulu, Finland

${ }^{8}$ Cancer and Translational Medicine Research Unit, Medical Research Center, University of Oulu and Oulu University Hospital, Oulu, Finland

${ }^{9}$ Department of Pathology, HUSLAB, HUS Diagnostic Center, Helsinki, Finland

${ }^{10}$ Applied Tumour Genomics Research Program, Research Programs Unit, University

of Helsinki and Helsinki University Hospital, Helsinki, Finland

${ }^{11}$ The Division of Digestive Surgery and Urology, Turku University Hospital, Turku, Finland

${ }^{12}$ Department of Surgery, Central Finland Central Hospital, Jyväskylä, Finland

${ }^{13}$ Upper Gastrointestinal Surgery, Department of Molecular Medicine and Surgery, Karolinska Institutet and Karolinska University Hospital, Stockholm, Sweden

Contributors JHK contributed to study idea. HKS, JR, JS, VT, TT, TR, AV, PO, MP, AK, RK, TJK, V-MP, AR, SL, ES and JHK involved in conceptualisation and design of the study. JR, JS, VT, TT, TR, AV, PO, MP, AK, RK, TJK, V-MP, AR, SL, ES and JHK involved in data collection and tools development. MP and JHK obtained permissions. JHK obtained funding. PO and JHK involved in statistical analysis. HKS, JR, JS, VT, TT, TR, AV, PO, MP, AK, RK, TJK, V-MP, AR, SL, ES and JHK involved in interpretation of data. HKS drafted the manuscript. JR, JS, VT, TT, TR, AV, PO, MP, AK, RK, TJK, V-MP, AR, SL, ES and JHK critically revised for intellectual content and accepted submitted version. JHK was a guarantor.

Funding This work is supported by research grants from the Sigrid Jusélius Foundation (Sigrid Juséliuksen Säätiö), The Finnish Cancer Foundation (Syöpäsäätiö), Päivikki and Sakari Sohlberg Foundation and Orion Research Foundation (Orionin Tutkimussäätiö).

Disclaimer The funding sources have no role in the design and conduct of the study; collection, management, analysis, and interpretation of the data; preparation, review, or approval of the manuscript; or decision to submit the study protocol for publication.

Competing interests None declared.

Patient and public involvement Patients and/or the public were not involved in the design, or conduct, or reporting, or dissemination plans of this research.
Patient consent for publication Not required.

Ethics approval The study has been approved by the ethical committee in Northern Ostrobothnia, and governmental agencies and hospital districts involved in the study. ${ }^{20}$ Due to the retrospective nature of this study, it is not required by Finnish law, nor reasonable, to obtain individual consent from patients included in the registries.

Provenance and peer review Not commissioned; externally peer reviewed.

Data availability statement Data are available upon reasonable request. All data from FINEGO presented in this article are stored by the research group on safe servers at University of Oulu, Finland, and handled confidentially. Currently, only the research team has access to the data. Researchers interested in collaboration, for example joint efforts combining the dataset with other population-based studies, are welcome to contact Joonas Kauppila (joonas.kauppila@oulu.fi), principal investigator.

Open access This is an open access article distributed in accordance with the Creative Commons Attribution Non Commercial (CC BY-NC 4.0) license, which permits others to distribute, remix, adapt, build upon this work non-commercially, and license their derivative works on different terms, provided the original work is properly cited, appropriate credit is given, any changes made indicated, and the use is non-commercial. See: http://creativecommons.org/licenses/by-nc/4.0/.

ORCID iD

Joonas H Kauppila http://orcid.org/0000-0001-6740-3726

\section{REFERENCES}

1 Anderson LA, Tavilla A, Brenner H, et al. Survival for oesophageal, stomach and small intestine cancers in Europe 1999-2007: results from EUROCARE-5. Eur J Cancer 2015;51:2144-57.

2 Rustgi AK, El-Serag HB. Esophageal carcinoma. N Engl J Med 2014;371:2499-509.

3 Global Burden of Disease Cancer Collaboration, Fitzmaurice C, Abate D, et al. Global, regional, and National cancer incidence, mortality, years of life lost, years lived with disability, and DisabilityAdjusted life-years for 29 cancer groups, 1990 to 2017: a systematic analysis for the global burden of disease study. JAMA Oncol 2019;5:1749.

4 Cancer statisticsFinnish cancer registry. Available: https:// cancerregistry.fi/statistics/cancer-statistics/ [Accessed 18 Aug 2020].

5 Edgren G, Adami H-O, Weiderpass E, et al. A global assessment of the oesophageal adenocarcinoma epidemic. Gut 2013;62:1406-14.

6 Arnold M, Laversanne M, Brown LM, et al. Predicting the future burden of esophageal cancer by histological subtype: international trends in incidence up to 2030. Am J Gastroenterol 2017;112:1247-55

7 Sjoquist KM, Burmeister BH, Smithers BM, et al. Survival after neoadjuvant chemotherapy or chemoradiotherapy for resectable oesophageal carcinoma: an updated meta-analysis. Lancet Oncol 2011;12:681-92.

8 Schmidt HM, Gisbertz SS, Moons J, et al. Defining benchmarks for transthoracic esophagectomy: a multicenter analysis of total minimally invasive esophagectomy in low risk patients. Ann Surg 2017;266:814-21.

9 Kauppila JH, Helminen O, Kytö V, et al. Short-Term outcomes following minimally invasive and open esophagectomy: a population-based study from Finland and Sweden. Ann Surg Oncol 2018;25:326-32.

10 Bouchard P, Molina J-C, Cools-Lartigue J, et al. Endoscopic submucosal dissection for esophageal adenocarcinoma: a North American perspective. J Gastrointest Surg 2019;23:1087-94.

11 Pech O, May A, Manner H, et al. Long-Term efficacy and safety of endoscopic resection for patients with mucosal adenocarcinoma of the esophagus. Gastroenterology 2014;146:652-60. e1.

12 Finks JF, Osborne NH, Birkmeyer JD. Trends in hospital volume and operative mortality for high-risk surgery. N Engl J Med 2011;364:2128-37.

13 Kauppila JH, Wahlin K, Lagergren P, et al. University hospital status and surgeon volume and risk of reoperation following surgery for esophageal cancer. Eur J Surg Oncol 2018;44:632-7.

14 Derogar M, Sadr-Azodi O, Johar A, et al. Hospital and surgeon volume in relation to survival after esophageal cancer surgery in a population-based study. J Clin Oncol 2013;31:551-7.

15 Markar SR, Lagergren J. Surgical and surgeon-related factors related to long-term survival in esophageal cancer: a review. Ann Surg Oncol 2020;27:718-23. 
16 Luketich JD, Pennathur A, Franchetti Y, et al. Minimally invasive esophagectomy: results of a prospective phase II multicenter trialthe eastern cooperative Oncology Group (E2202) study. Ann Surg 2015;261:702-7-7.

17 Yibulayin W, Abulizi S, Lv H, et al. Minimally invasive oesophagectomy versus open esophagectomy for resectable esophageal cancer: a meta-analysis. World J Surg Oncol 2016;14:304-7.

18 Gottlieb-Vedi E, Kauppila JH, Malietzis G, et al. Long-term survival in esophageal cancer after minimally invasive compared to open esophagectomy: a systematic review and meta-analysis. Ann Surg 2019;270:1005-17.

19 Straatman J, van der Wielen N, Cuesta MA, et al. Minimally invasive versus open esophageal resection: three-year follow-up of the previously reported randomized controlled trial: the time trial. Ann Surg 2017;266:232-6.

20 Kauppila JH, Ohtonen P, Karttunen TJ, et al. Finnish national Esophago-Gastric cancer cohort (FINEGO) for studying outcomes after oesophageal and gastric cancer surgery: a protocol for a retrospective, population-based, nationwide cohort study in Finland. BMJ Open 2019;9:e024094-024094.

21 Leinonen MK, Miettinen J, Heikkinen S, et al. Quality measures of the population-based Finnish cancer registry indicate sound data quality for solid malignant tumours. Eur J Cancer 2017;77:31-9.
22 Brusselaers N, Lagergren J. The Charlson comorbidity index in registry-based research. Methods Inf Med 2017;56:401-6.

23 Lindblad M, Ye W, Lindgren A, et al. Disparities in the classification of esophageal and cardia adenocarcinomas and their influence on reported incidence rates. Ann Surg 2006;243:479-85.

24 Rice TW, Rusch VW, Apperson-Hansen C, et al. Worldwide esophageal cancer collaboration. Dis Esophagus 2009;22:1-8.

25 Low DE, Alderson D, Cecconello I, et al. International consensus on standardization of data collection for complications associated with esophagectomy: esophagectomy complications consensus group (ECCG). Ann Surg 2015;262:286-94.

26 Kauppila JH, Mattsson F, Brusselaers N, et al. Prognosis of oesophageal adenocarcinoma and squamous cell carcinoma following surgery and NO surgery in a nationwide Swedish cohort study. BMJ Open 2018;8:e021495.

27 Quality description: causes of death 2016Statistics Finland. Available: https://www.stat.fi/til/ksyyt/2016/ksyyt_2016_2017-12-29_ laa_001_en.html [Accessed 18 Aug 2020].

28 van Hagen P, Hulshof MCCM, van Lanschot JJB, et al. Preoperative chemoradiotherapy for esophageal or junctional cancer. N Engl $J$ Med 2012;366:2074-84.

29 Low DE, Kuppusamy MK, Alderson D, et al. Benchmarking complications associated with esophagectomy. Ann Surg 2019;269:291-8 